\title{
Identification of New Brain Targets by Neuro-Imaging Technology for Treatment of Neurocardiac Disorders
}

\author{
Ram B Singh ${ }^{1}$, V I Torshin ${ }^{2}$, OA Bawareed ${ }^{2}$, NS Verma ${ }^{3}$, M Fabian ${ }^{4}$, S Wichansawakun ${ }^{5}$, \\ A Chladekova ${ }^{6}, V^{\text {V Mojto }}{ }^{6}$ and Dr Kumar Kartikey ${ }^{7}$ \\ ${ }^{1}$ Halberg Hospital and Research Institute, India
}

${ }^{2}$ People's Friendship University of Russia, Russia

${ }^{3}$ Department of Physiology, India

${ }^{4}$ Magnetic Resonance Dr. Magnet - Kramare, Slovakia

${ }^{5}$ Department of Internal Medicine, Thailand

${ }^{6} 3^{\text {rd }}$ Internal Clinic Faculty of Medicine Comenius University, Slovakia

${ }^{7}$ Halberg Hospital and Research Institute, Moradabad, India

*Corresponding author: Dr Mojto, $3^{\text {rd }}$ Internal Clinnic Faculty of Medicine, Comenius University, Bratislava, Slovakia

\section{ARTICLE INFO}

Received: 豐 January 21, 2019

Published: 幽 February 04, 2019

Citation: Ram B Singh, V I Torshin,, OA Bawareed, NS Verma, M Fabian, S Wichansawakun, A C, V Mojto, Dr Kumar K. Identification of New Brain Targets by Neuro-Imaging Technology for Treatment of Neurocardiac Disorders. Biomed J Sci \& Tech Res 14(1)-2019. BJSTR. MS.ID.002484.

Keywords: Mental Stress; Anxiety Disorders; Depression; Emotional Stress; Heart Attack

Abbreviations: fMRI: Functional Magnetic Resonance Imaging; 3D-Echo: 3-Dimensional Echocardiography; ACS: Acute Coronary Syndrome; Cmds: Cardiometabolic Diseases; Cvds: Cardiovascular Diseases; PET: Positron Emission Tomography; Ce A: Central Amygdala, BA: Basal Amygdala; LA: Lateral Amygdala; BA: Basal Amygdala; Lfps: Local Field Potentials; BOLD: Blood-OxygenLevel-Dependent; DTI: Diffusion Tensor Imaging; CS: Conditioned Stimuli, US: Unconditioned Stimuli; GAD: Generalized Anxiety Disorders; CRF: Corticotropin-Releasing Factor

\author{
ABSTRACT
}

Recently, neuroimaging technology for study of neuro-cardiac physiology and mental and spiritual health has gained interest, in relation to cardiovascular function and other body systems. Advances in functional magnetic resonance imaging(fMRI) and nuclear imaging techniques appear to be quite important in the diagnosis of neurological diseases, psychological disorders, apart from extended knowledge of pathophysiology of atherosclerosis and vulnerable plaques. 3-dimensional echocardiography (3D-Echo.) and multi-detector-row computed tomography are important technological advances that have improved our capability to know more about cardiovascular diseases (CVDs). Rapid changes in environmental factors have predisposed the people to psychosocial stress on a daily basis, in both developed and developing countries. Heavy workloads, job insecurity, living in relative poverty, and competition in business have been associated with increases in mental stress, which in turn can lead to chronic anxiety disorders, such as anxiety, aggression, type A behavior and depression. Apart from increases in psychological burden, chronic stress may also be associated with an increased risk of CVDs; atherosclerosis, hypertension, heart failure, and diabetes. Epidemiological studies indicate that subjects with increased stress perception have a substantially higher prevalence of CVDs than controls. Chronic and acute emotional stress and sleep disturbances can damage certain areas of the brain, such as amygdala, hippocampus, hypothalamus, in particular circadian clock that are known to predispose inflammation in the arteries, leading to atherosclerosis. Recent evidence, has demonstrated that chronic anxiety disorders such as depression, may be a risk factor of CVDs and diabetes which may be associated with tremors in the brain areas concerned with related physiological functions. The autonomic nervous system involves the sympathetic activity, which brings about increased catecholamines and cortisol, oxidative stress, hyperglycemia with a rise in free fatty acids, which may be a pro-inflammatory state. Increased parasympathetic activity related to vagal nerve stimulation may be associated with increased release of acetylcholine, a precursor of nitric oxide which is anti-inflammatory. Acetylcholine is the principal vagal neurotransmitter, which is known to attenuate the release of cytokines, tumor necrosis factor (TNF), interleukin (IL)-1 , IL-6 and IL-18, without any adverse effect on the anti-inflammatory cytokine IL-10, in lipopolysaccharide-stimulated human macrophage cultures. A regular practice of spiritual methods such as yoga, meditation, and active prayer can decrease the risk of CVDs, metabolic syndrome as well as pro-inflammatory cytokines. Circadian alteration in neuronal metabolism in the hypothalamus and cortex, in particular medial temporal lobe have been observed in morning compared to evening. The above non-drug measures as well as therapeutic agents can improve autonomic functions, possibly due to increased parasympathetic activity, which can be visualized as thickness in the cortex, in certain areas of brain by neuroimaging studies. These new imaging markers may be used as new targets of therapy for advances in therapy of various diseases. 


\section{Introduction}

Budha,s Eight Fold Path aims to modulate the mentaland spiritual health, although, the relation of mind, brain and body functions, was known in ancient India for the last 5000 years, as mentioned in the ancient scripture - Bhagwatgita (3000 BCE). Recently, World Heart Journal has reviewed the relation of neuro-cardiac physiology with mental and spiritual health and the role of neuro-cardiovascularimaging in relation to cardiovascular function and other body systems [1-3]. The reviews are based on evidence published in the world class journals between 2016 to 2018 [4-8]. Advances in new technologies such as functional magnetic resonance imaging(fMRI) and nuclear imaging techniques have become quite important in the diagnosis of neurological diseases, psychological disorders, apart from study of pathophysiology of atherosclerosis and vulnerable plaques. 3-dimensional echocardiography (3D-Echo.) and multidetector-row computed tomography have improved our capability to know more about valvular heart disease as well as acute coronary syndrome (ACS) [2,4]. Rapid increase in industrialization and urbanization during the last one century have been associated, with marked changes in diet and lifestyle factors and an increase in psychosocial stress resulting in to emergence of cardiometabolic diseases (CMDs); cardiovascular diseases(CVDs) and diabetes [13]. Further evidence also indicates that a majority of individuals in developed and developing countries experience psychosocial stress on a daily basis as a result of urbanization and industrialization and religious services may be protective [2-5]. Heavy workloads, job insecurity, living in relative poverty, and competition in business have been associated with increases in mental stress, which in turn can lead to chronic anxiety disorders such as depression [9-12].

Apart from increases in psychological burden; chronic stress may also be associated with an increased risk of CVDs; atherosclerosis, hypertension, and heart failure, and of diabetes [1-4]. These subjects with increased stress perception have a substantially higher prevalence of myocardial infarction than controls as revealed in the INTERHEART Study [11,12]. A recent study reported that subjects with heightened metabolic activity in the amygdala (an area of the brain linked to emotional stress) was associated with a greater risk of heart disease and stroke [4]. Other studies indicated that fMRI is used to correlate most of the psychological disorder as well as CVDs [13-18]. These studies indicate that advancements in therapies may be achieved, via new targets obtained by neuro-cardic imaging. This review aims to examine the available evidence on the relation of neuro-cardiac imaging markers with chronic anxiety disorders and CVDs.

\section{Physiological Imaging Of Brain}

In general, neurological dysfunction in neuronal biology do not cause significant correlations with visible alterations in the morphology of the cells. However, the contrast taken for the identification of abnormalities in the physiopathology or biology can define the accuracy of an imaging modality regarding it performance. Recently, more sensitive imaging methods for identification of alterations in brain function have been developed. In view of the varieties of available imaging modalities, it is clear that multiple capabilities of the various kinds of radiation are used in our practice [19]. The positron emission tomography (PET) and functional magnetic resonance imaging (fMRI) are the commonly used and most promising molecular imaging methods for the identification of alterations in brain functioning. In view of the difficulties noted during developing the specific contrast agents for MRI, methods for fMRI use was identified and therefore the success of fMRI is mainly because of the presence of an endogenous contrast agent in human body which has been found to have high sensitivity. Interestingly, fMRI can provide exceptional features which increase its performance [20].

The blood of adults contains about $750 \mathrm{~g}$ of hemoglobin that is rich in iron, which cannot be achieved in any endogenous agent that can act as contrast. The iron in deoxy- hemoglobin acts as a contrast agent in the fMRI technique. In presence of oxidation, hemoglobin may loose its contrast enhancing nature. In the deoxygenated hemoglobin, the deoxyhemoglobin/oxyhemoglobin ratio defines the intensity of signals because iron is paramagnetic due to its four unpaired electrons [21]. It is similar to gadolinium in the common MRI contrast agents. The neural activity as well as spiking of neurons, can occur due to rise in the uptake of glutamate in astrocytes which may be under influence of blood oxygen level dependent effect $[20,21]$. In the high performance of fMRI, many features contribute to clearance of physiopathology via physiological imaging of neurons as given in Table 1. There are several neuro-psychiatric dysfunctions in which brain imaging has been used to correlate mental diseases with images of brain areas such as in schizophrenia, depression, anxiety disorders etc [21-30]. The amygdalar activity which is known to transform stressful events into anxiety and and fear extinction, have also been studied via fMRI which may be used as new targets for advances in therapy [31-33].

Table 1: Special features for better performance of fMRI.

1. Clinical convenience by which fMRI is used with the standard MRI scanners and does not need any exogenous contrast agents.

2. The contrast agent in the body tissues has its own function that responds to stimuli.

3. fMRI do not need the use of radioactive probe but it can be easily repeated and thus commonly used in longitudinal studies.

4. Its easy to study the changes in the morphology of brain which may influence a certain region of brain, as well as to other brain region connectivity.

5. Contrast is achieved due to the estimation of iron in hemoglobin; unoxidized hemoglobin, that is high in the brain tissues.

\section{Biochemical Pathways for Nutrition and Brain Dysfunction}

Serotonin deficiency has been commonly observed among patients with mood disorders [33,34]. Within the central nervous system, there are diffused networks made by serotonin system 
which play significant role in the regulation of mood and cognition [35]. Acute or chronic change in tryptophan level, by depletion or supplementation, may be an experimental procedure for modifying peripheral and central serotonin concentrations, that determine mood and behavior [33]. Western diets, particularly a diet low in \-3 fatty acids, flavonoids as well as essential and nonessential amino acids, are pro-inflammatory and may increase the risk of CVDs, diabetes, as well as degenerative diseases of the brain by causing oxidative stress, leading to fibrosis and inflammation in various organs [34-36]. Many protein-based foods, including meats, dairy products, dry fruits, and seeds, are rich in tryptophan, which is an essential amino acid. High-glycemic index and high-glycemic load meals also increase the availability of tryptophan [35]. A balance between dietary intake of tryptophan and its removal from the plasma as a part of its essential role in protein biosynthesis may be important determinants of plasma concentrations of tryptophan [35].

It is a precursor for a number of metabolites, most notably kynurenine and the neurotransmitter serotonin, which is protective against depression [35]. The second most prevalent metabolic pathway of tryptophan availability after protein synthesis may be the synthesis of kynurenine, which accounts for approximately $90 \%$ of tryptophan metabolism [35]. There is strong evidence implicating the kynurenines (precursor of kyruneric acid, an antagonist at glutamate, ionotropic receptors) to be important in the pathogenesis of behavioral and cognitive symptoms of neuropsychiatric diseases [35]. It is the plasma amino acid pattern, in particular, the plasma /tryptophan ratio which provides information about the composition of diet via food induced alterations. Thus food quality may induce an increase or decrease in brain tryptophan levels, which is important in the synthesis of serotonin. A carbohydrate-rich, low protein meal stimulates insulin secretion that may diminish plas- ma concentrations of the amino acids. It is known that many amino acids; leucine, isoleucine and valine compete with tryptophan for transport into the brain. It is clear that increasing tryptophan's flux across the blood-brain barrier and its brain concentrations. However, a high-protein meal contributes too much of amino acids to the blood stream than of the relatively-scarce tryptophan, which diminishes tryptophan's entry into the brain. Thus, the composition of the diet appears to be important in the physiology and metabolism of amino acids and availability of serotonin which are known to influence various targets of therapy seen via brain imaging.

\section{The Gut-Brain Axis and Emotional Health}

The gut-brain axis is a bi-directional system between the brain and gut, which may be linking emotional and cognitive centers of the brain with peripheral functioning of the gut. The influence of the gut microbiome on behavior is becoming increasingly evident, as is the extension to tryptophan and serotonin, which poses the possibility that alterations in the gut may be important in the pathophysiology of neuropsychiatric disorders. Further evidence indicates that low brain serotonin concentrations are associated with poor memory and depressed mood, which may be a precursor of depression. Manipulating tryptophan in diet and plasma may influence mood and cognition, having a possible influence on the gut-brain axis, because serotonin synthesis also occurs in gut neurons [35]. Therefore, dietary factors as well as gut dysfunctions can influence brain dysfunctions and emotional health, which may be observed via brain imaging. In a meta-analysis involving 55 PET and activation of fMRI studies that produced 761 individual peaks, the authors examined the association of anxiety disorders in apparently healthy participants with activation of certain areas of the brain [14]. The coordinates with peak activation were altered into a standard space and plotted onto canonical 3-D brain renderings. The brain was divided into 20 non-overlapping regions.

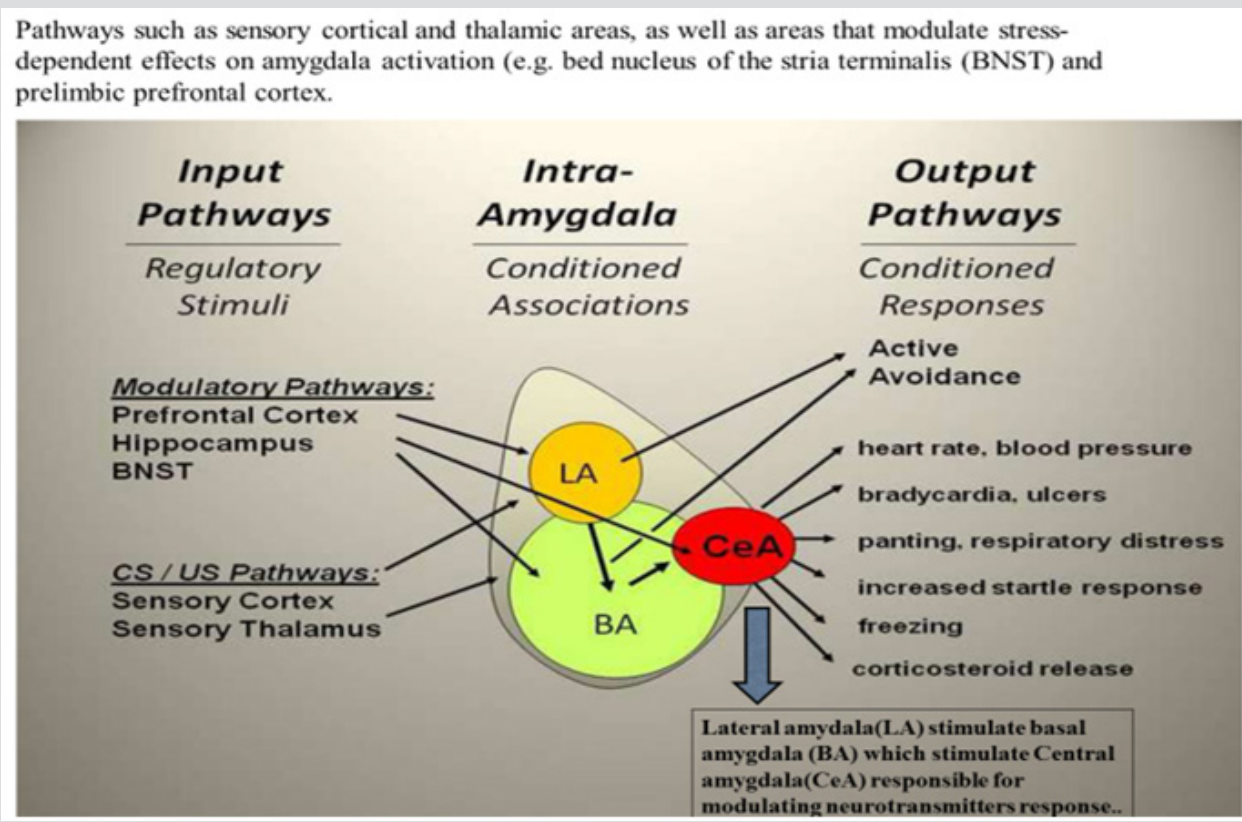

Figure 1: Amygdala modulating the fear response in humans (modified from Ressler, 2010 [16]). 
Across any of the single emotions such as positive, negative, happiness, fear, anger, sadness, disgust, each region showed a characteristic response to different induction methods that could be visual, auditory, recall/imagery depending on emotional tasks with and without cognitive demand. Table 2 shows the findings suggesting that separate brain regions are involved in different aspects of emotions, which may predispose to a greater risk of CVDs $[14,16]$. Modified from Phan et al. [14]. In several brain imaging studies, fearful stimuli, including frightening faces, fear-inducing images, and fear-conditioned cues, were reported to cause amygdala activation [14-18]. Thus increased activity of amygdala, in response to fearful stimuli was found in 25 studies examining the functional brain structure of emotion, while 4 studies found activation to positive stimuli [16]. An experimental study in rats indicated that increase in the plasticity in in certain areas of brain, e g, prefrontal cortex (PFC) and/or hippocampus, can increase the therapeutic effects of treatment of cognition [15]. The results showed that administration of a novel magnesium therapy (magnesium-l-threonate [MgT]) increased brain magnesium, which was associated with increase in hippocampal synaptic plasticity as well as in learning and memory function in rats [15]. Treatment with MgT enhanced retention of the extinction of fear memory, without enhancing, impairing, or erasing the original fear memory [15] (Figure 1).

There are at least 13 different sub-nuclei in the amygdala, which are may be seen in Figure 2, including the central amygdala (Ce A), the basal (BA) and lateral (LA) amygdala nuclei $[14,16]$. The central amydala has a influence on the fear response, including regulation of the cortisol release via the paraventricular nucleus of the hypothalamus, known to regulate subcellular remodeling in the cardiomyocyte and sarcolemma. The central amygdala also enhances the startle response through the midbrain, and stimulate the autonomic nervous system via lateral hypothalamus [16]. If the central amygdala is damaged, it eliminates fear-conditioned responses, for example, fear-potentiated startle and freezing. Hence, central amygdala may be considered as the primary output or effector region [16].Within the amygdala, the learning is accomplished by lateral amygdala and basal amygdala (BA), in particular, the lateral amygdala gets signals from visual and auditory areas (Table 2). New therapies may be developed to target new imaging markers obtained by neuroimaging.



Figure 2: Mental dysfunction-induced increased amygdaler activity in humans leading to systemic inflammation (modified from Tawakol et al. [4]).

Table 2: Effects on neuro-anatomical region activation via concerned emotions.

\begin{tabular}{|c|c|c|}
\hline No & Neuronal region & Mental dysfunction \\
\hline 1. & Medial frontal cortex & Emotional processing \\
\hline 2. & Amygdala & Sadness \\
\hline 3. & Subcallosal cingulate & Emotional induction by visual stimuli \\
\hline 4. & Occipital cortex and amygdala & Induction by emotional recall imagery \\
\hline 5. & Anterior cingulate and insula & Emotional task with cognitive demand \\
\hline 6. & Anterior cingulate and insula & \\
\hline
\end{tabular}




\section{Functional (MRI/PET) Imaging of Brain in Schizophrenia}

fMRI has been widely used to study the operational organization of the human brain, by finding out the relationship between the measured fMRI signal and the underlying neural activity. Here we describe the simultaneous intra-cortical recordings of neural signals and fMRI responses. In a recent study in monkeys, local field potentials (LFPs), single- and multi-unit spiking activity with highly spatio-temporally resolved blood-oxygen-level-dependent (BOLD) fMRI responses were examined from the visual cortex [21] LFPs were observed to be the largest magnitude changes, which at recording sites characterized by transient responses were the only signal that significantly correlated with the hemodynamic response. The impulse response of the neurovascular system is both animal- and site-specific, and that LFPs yield a better estimate of BOLD responses than the multi-unit responses as revealed by linear systems analysis on a trial-by-trial basis. The results indicate that the BOLD contrast mechanism reflects the input and intracortical processing of a given area rather than its spiking output.

Majority of the vascular and neuronal diseases, of the brain can be easily diagnosed which could be helpful in appropriate therapies. Apart from diagnosis, fMRIs may be used prior to surgeries of the brain, to educate the surgeon a subjective organ and tissue mapping of visual and clinical functions of the subject. Recently, fMRI has been found to have important role in brain imaging research related neuro-psychiatric and cardiovascular diseases [4,5]. In 2001, Shenton et al reported, that MRI and fMRI examination in patients with schizophrenia, indicate a loss of volume of brain tissue, in particular an increase of the size of ventricles, which could be one an important result of MRI examination [22]. Kindermann et al were the first to show that fMRI in patients with schizophrenia and deficient cognition may be characterized with neural substrate in the temporal cortex as well as in dorsolateral prefrontal cortex [23]. However, there is greater focus now on research in patients with schizophrenia related to socio-emotional processing in a resting state to find out changes that could be independent of task in the connectivity of networks at prefrontal and temporal regions [24].

In schizophrenia, fMRI may reveal altered functional as well as structural prefrontal-temporal communication via examination using diffusion tensor imaging (DTI) to detect loss of myelin of the white matter $[25,26]$.The molecular processes in the brain examined via fMRI include imaging of genetics functions e.g., a certain SNiP which can influence dopamine release in the frontal cortex on activation of neurons [27]. It seems that via use of MRS, the level of only few neurotransmitters can be assessed. However, imaging genetic studies may exclude epigenetic changes, but PET examines several molecular targets of tissues of brain by a t direct assessment. Other benefits of MRS studies include imaging studies of genetic dysfunctions, by combining of PET and fMRI which may give an opportunity for direct comprehensive examination of the functioning of each region of brain, and interactions of the areas in the whole brain. It is also possible that examination of the glutamate in the prefrontal cortex among schizophrenia patients, MRS could be an important help for solving the mechanism of cognitive deficits in schizophrenia [28]. In Alzheimer,s disease, PET screening provides disturbed metabolism and function of the neurons [29].

In a clinical study among 5 healthy volunteers and 6 patients with neurological problems, 18F]-2-Fluoro-A85380 uptake was clearly visualized and maximum target-to-background ratio uptake values corrected for the background activity of the tracer showed specific tracer uptake in the arterial walls [30]. In the descending aorta, significantly higher uptake values were found. Comparison between volunteers and patients showed significant differences, with lower [18F]-2-Fluoro-A85380 uptake in the patient group when comparing single arterial territories but not when all arterial territories were pooled together. It is possible that [18F]2-Fluoro-A85380 can provide specific information on the nAChR distribution in human arteries[30]. Vascular nAChR density seems to be lower in patients with Parkinson's disease or multiple system atrophy. Once confirmed in larger study populations and in the experimental setting, this approach might provide insights into the pathogenic role of nAChRs in the human vasculature. It seems that advancements in the techniques of the imaging of brain, such as fMRI and PET screening, have enhanced our knowledge in understanding the molecular structure and physiological function of the brain in humans. which has been considered quite complex earlier. Restrictions in funds and concerns related with safety, have reduced the further progress in the getting new tracers in PET studies.

However, functional MRI hemodynamic based studies, continue to advance research and progress in neuroimaging. Functional MRI has become the major modality for neuroimaging among patients for diagnosis of diseases and possibly targeted therapy. The radio tracers for molecular imaging; such as PET, high light the basis for greater knowledge of molecular mechanisms in brain tissues. It also helps in selecting the procedures for safety concerns of each therapy. There is going to be a new era, due to use of BOLD fMRI measuring brain function with PET radiolabeled reporters which can be useful in mapping the more difficult molecular targets. Such targets could be distribution and function of receptors synergistics which may further enhance our insight into brain function [27-30]. A new era may also be expected in the advancement of therapies due to these new imaging markers for targeted therapy.

\section{Functional (MRI/PET) Imaging of Brain in Depression}

There is evidence that depression and other mood disorders have become important precursors of CMDs and other chronic diseases [4-10]. Therefore, it is important to understand the neurological mechanisms using functional imaging of brain via fMRI and PET screening. Previous studies also showed that emotions can influence the amygdala and the neuropeptide $\mathrm{S}$ system, which 
is capable of interfering with mechanisms in the amygdala that transform stressful events into anxiety and impaired fear extinction $[31,32]$. The functional neuroanatomy of depression reveals that ventromedial and dorsolateral sectors of the prefrontal cortex are critical neural substrates with distinct functional contributions [33]. The putative roles of the ventromedial and dorsolateral prefrontal cortex in depression include functional alterations of neural circuits among individuals with depression. Most patients with major depression also show aberrant neural activity in the hippocampus, striatum, prefrontal cortex, anterior cingulate cortex, and the thalamus, among many other structures [33]. Functional neuroanatomical studies have shown that neural activity of the hippocampus can also predict a subsequent reduction in activation of the dorsolateral prefrontal cortex in patients with depression. Further evidence indicates that depressive symptoms are associated with abnormally low levels of dorsolateral prefrontal cortex activity.

Treatment of depression in response to behavioral therapy or to antidepressant medications may result in increased activity in this brain region [33]. Since the dorsolateral prefrontal cortex is linked to executive function, it may help explain some of the observed changes in working memory, cognitive flexibility, attention, and motivation among people who suffer from depression. This review also emphasized that an atrophy of numerous structures within the central nervous system may be observed among subjects suffering from depression [33]. Neuroscientists have reported that the amygdala, hippocampus, prefrontal cortex, striatum, nucleus accumbens, and the cerebellum are some of the many structures of the brain that are smaller in size among patients with depression, as compared with those of typical, healthy individuals, which may be due to inflammation leading to fibrosis [14,33-35]. It may be a principal locus for associations between previously neutral conditioned stimuli (CS) and aversive, e.g., shock or trauma, unconditioned stimuli (US), resulting in the acquisition of conditioned fear. The BA receives some direct CS and US pathways, but is also a target area for further processing of information from the LA prior to sending CS-US information to the CeA [14-16]. There is a need to examine the effects of therapies on these imaging targets for advancement of new therapies [36,37].

\section{Functional Imaging in Generalised Anxiety Disorders}

Recent studies propose that generalized anxiety disorders(GAD) or chronic stress and sustained threats are inter-related and the measurable units of analysis may provide important information, that may be critical for designing effective targeted therapies [38]. Many subjects with acute stress may have repeated triggering, resulting in to chronic stress, due to chronic maladaptive stress responses causing psychological disorders. There may be involvement of biological systems; autonomic, neuroendocrine, and psychological that may be triggered by actual or perceived or threat leading to GAD. GAD as well as any long term anxiety may be cause increased concentrations of global DNA methylation and changes in the expression of epigenetic and gene of interleukin- 6 [39]. On the other hand, alterations in amygdala-prefrontal functional connectivity, in particular fronto-limbic structures, can account for increased anxiety and autonomic dysregulation in GAD [40]. This study based on neuroimaging, assessed the association of the connectivity between both prefrontal cortex and amygdala and autonomic heart rate variability in patients suffering from GAD pre- and post-perseverative cognition task [40]. The subjects with GAD showed lower connectivity between the prefrontal cortex and amygdala at baseline. The prefrontal-amygdala connectivity showed increase in the GAD group, but controls had decrease in this area after the preservative cognition task (at a trend level).

Interestingly, after the perseverative cognition task, decline in heart rate variability (increase in autonomic activation) was predicted by reduced resting-state functional connectivity between the left amygdala-subgenual cingulate cortex and between the right amygdala-caudate nucleus [41]. These results indicate new finding on specific neural modulation of differential psycho-physiological responses between GAD and apparently healthy controls. In both adults and adolescents with GAD, the disturbed coordination of brain activity has been proposed as one of the core neurobiological manifestation of GAD. In a review, neural structures, functioning and connectivity in GAD as well as interaction with neuroendocrine systems has been reported [42]. The decline in resting-state functional connectivity, between the amygdala and prefrontal cortex, suggest differences between GAD and healthy control subjects [41,42]. In structural and functional neuroimaging, differences exist in frontolimbic areas as well as in a downstream projection from the amygdala: the anterior cingulate cortex [43]. The results indicate that across adolescence and adulthood, decreased connectivity between the amygdala and prefrontal cortex is associated with the diagnosis of GAD [44-47].

It is proposed that the prefrontal cortex appears to be critical for the effective regulation of emotion, particularly ventromedial regions that appear to control emotions during anxiety. In a metaanalysis, it has been estimated that depth of fear is limited by the mind which may have negative effects due to anxiety [46]. Heart rate and heart rate variability in panic, social anxiety, obsessive, Ä00EC; compulsive, and GAD at baseline may be under influence of relaxation and hyperventilation [48]. The ventrolateral prefrontal cortex activity has been reported to rise, possibly by enhancing control on limbic structures, after therapeutic intervention in subjects with GAD [47]. Sustained threats may be associated with dysfunction of molecular components, for example, hypothalamic- pituitary-adrenal axis, corticotropin-releasing factor (CRF); adrenocorticotropic hormone and paraventricular nucleusn [48]. These molecular components related to sustained threat are also component of the neuroendocrine system, that controls the response caused by stress. It has been proposed that neuroendocrine dysregulation may contribute anxiety, in particular, when there is a state of threat or anxiety. It has been observed that 
corticotrophin releasing factor is released from the paraventricular nucleus of the hypothalamus into the primary capillary plexus. It is part of hypothalamo-hypophyseal portal system that stimulate the anterior pituitary to synthesize pro-opiomelancortin into the blood. In the adrenal glands, adrenocorticotropic hormone in the blood activates the synthesis and release of cortisol.

Table 3: Research domain areas in the brain and criteria of sustained threat.

\begin{tabular}{|c|c|c|c|}
\hline Cells & Molecules & Circuits, physiology & Behavior \\
\hline $\begin{array}{c}\text { Hippocampal } \\
\text { Microglia } \\
\text { Prefrontal }\end{array}$ & $\begin{array}{c}\text { ACTH CRF HPA-axis hormones } \\
\text { Note. } \mathrm{ACTH}=\text { (adrenocorticotropic } \\
\text { hormone); } \mathrm{CRF}=\text { (corticotropin releasing } \\
\text { factor); HPA= hypothalamic-pituitary- } \\
\text { adrenal); }\end{array}$ & $\begin{array}{c}\text { Attention network Dysregulation of amygdala } \\
\text { reactivity Dysregulation of cingulate reactivity } \\
\text { Habit systems Hypothalamic nuclei, PVT } \\
\text { (paraventricular thalamic nucleus)., Vigilance } \\
\text { network Dysregulated HPA Axis Error-related } \\
\text { negativity }\end{array}$ & $\begin{array}{c}\text { Decrease in appetitive, Arousal } \\
\text { associated with anxiety. Attentional } \\
\text { bias to threat, reduced libido, } \\
\text { avoidance. Behavior with } \\
\text { helplessness, greater detection } \\
\text { of conflict, greater perseverative } \\
\text { behavior, Memory retrieval deficits } \\
\text { Punishment sensitivity }\end{array}$ \\
\hline
\end{tabular}

There is shut down of the hypothalamic-pituitary-adrenal axis, due to release of cortisol, or corticosterone, through the influence of the hypothalamus, pituitary, and hippocampus. In several studies, changes in the neuroendocrine system related to sustained threat; chronic activation of the system described above have been reported $[47,48]$ (Table 3). These neuroimaging targets can be used for development of new therapies in the treatment of diseases.

\section{Functional Imaging of Brain in Cardiovascular Diseases}

The details of atheroma in the arteries and various areas of brain may be examined by fMRI and PET screening which prompt us to establish a relation between neuronal physiology, with cardiovascular physiology and pathology [4-6]. Atherosclerosis and athero-thrombosis are major underlying causes of CVDs that are tenfold more common as vascular disease in diabetes. fMRI studies of brain and other parts of the body reported that chronic and acute emotional stress can damage certain areas of the brain causing tremors in these areas of brain predisposing systemic inflammation including inflammation in the arteries, leading to atherosclerosis $[4,6]$. Therefore, atherosclerosis may be considered as if it is a disease of the brain (Table 1) (Figures 1 \& 2). A landmark cohort study has confirmed this concept which included 293 subjects, aged 30 years or older, without known cardiovascular disorders, in whom amygdalar activity, bone-marrow activity, and arterial inflammation were examined fMRI[4]. After a median follow-up of 3.7 years, the results showed that increase in amygdalar activity examined by fMRI, was significantly associated with greater bonemarrow activity $(r=0.47, \mathrm{p}<0.0001)$, arterial inflammation ( $\mathrm{r}=0.49$, $\mathrm{p}<0.0001$ ), and increased risk of CVD events (standardized hazard ratio: $1.59,95 \%$ CI: $1.27-1.98, \mathrm{p}<0.0001)$.

The association between amygdalar activity and CVD events may have been mediated by increased bone-marrow activity and arterial inflammation. It was also observed in a separate crosssectional study, by psychometric analysis $(n=13)$, that amygdalar activity was significantly associated with arterial inflammation $(r=0.70, p=0.0083)$. The increase in the perception of stress was associated with increase in amygdalar activity ( $r=0.56, p=0.0485)$, arterial inflammation ( $\mathrm{r}=0.59, \mathrm{p}=0.0345)$, and C-reactive protein $(r=0.83, p=0.0210)$, which are risk factors of atherothrombosis
[13-16,1-5]. In the link of regional brain activity to subsequent CVDs, amygdalar activity independently and robustly predicted cardiovascular events [13]. Amygdalar activity is involved partly via a pathway that includes increased bone-marrow activity and arterial inflammation, which can be possibly modulated by neuronal omega-3 fatty acids, coenzyme Q10, and flavonoids. These findings provide novel insights into the mechanism through which emotional stressors can lead to CVDs, including atherosclerosis in human beings. The stress signaled in the amygdala is also linked to increased bone marrow activity and to inflammation in the arteries, leading to athero-thrombosis, which can cause heart attack and stroke [4]. A plausible biological mechanism could be that the amygdala triggers the bone marrow to produce extra white blood cells and increased inflammation, which then predisposes the arteries to develop atherosclerotic plaques, which can predispose to athero-thrombosis, resulting in CVDs [4].

It has been proposed that repeated osteocyte activity in the bones due to psychosocial stress, in particular when there is deficiency of omega-3 fatty acids, calcium, magnesium and coenzyme Q10, may predispose to inflammation, resulting in increased rate and severity of osteoporosis (Personal communication, Kumar Kartikey, Reema Singh and Sushav Singh) [1-3]. Western diet and modern lifestyles characterized by late-night sleep and deprivation of sleep can predispose to metabolic dysfunctions, resulting in increased vulnerability to emotional stress, inflammation, and risk of CVDs and diabetes [10-12]. Acute and chronic psychological stress, including affective disorders, are also known as risk factors for CVDs, which have been established from epidemiological, clinical and experimental studies [1-4,13-15]. Chronic psychosocial stress increases vulnerability to diet-related abdominal fat, oxidative stress, and metabolic risk, which are known to predispose to the triple burden of diseases $[13,35]$. Depression is a well-known risk factor of CVDs and diabetes, and can increase the risk of mortality [16-18].

Depression and other mental dysfunctions have made the prevention of CVDs quite challenging, as mentioned in a scientific statement of the International College of Cardiology [36]. Recent evidence for low-grade systemic pro-inflammatory activity in 
patients with posttraumatic stress disorder has been also observed, which may enhance arterial inflammation and plaque vulnerability, resulting in atherothrombosis via plaque rupture or plaque erosion [35].The above neuro-cardiac imaging markers should be used to develop new therapies.

\section{Physiological Imaging of Brain And Effects of Religious Services?}

The literature on religion and spirituality indicate their influence on mental health showing that religious beliefs may have some influence on health behavior and brain function $[3,49$ 51]. Examination of relationships with both positive and negative mental health outcomes, where positive outcomes include wellbeing, happiness, hope, optimism, and gratefulness have been studied under guidance of the late Dr. Franz Halberg [52-54]. The negative outcomes involve depression, suicide, anxiety, psychosis, substance abuse, delinquency/criminality, marital instability, and personality traits (positive and negative) are known to influence heart rate and blood pressure, indicating that they may also influence mental and physical health which may be protected by improved spiritual health and global health [54-56].Chronic variable mental dysfunction may activate the hematopoietic system, in particular stem cells, which may have individual differences in the peripheral immune system known to promote resilience depending upon susceptibility to social stress $[57,58]$. The mental stress response of the individual may be modulated by meditation, regular active prayer, and verbalization as well as by breathing exercises and moderate physical activity [3]. A recent cohort study by Li et al. included 74,534 participants with 13,537 reported deaths at follow-up [5]. There was an inverse association of attendance at religious services and adverse health outcomes, which may be due to CVDs and other chronic diseases that may be influenced by poor mental health. However, the study may have a few limitations in terms of generalization among other populations, such as men versus women (eastern women more religious than men) and subjects from various ethnicities with various religious beliefs, some of which are acknowledged by the authors.

The above landmark study is a major contribution demonstrating a favorable distribution of participants across the different categories of attendance at religious services, from almost never to more than once per week, with ample numbers for comparison in each category [5]. Particular strengths of the study relate to the large sample size and the excellent participation over time by the enrolled women, and hence the ability to test for the temporal association between the independent variables and the outcome variable at multiple time points. Time-varying covariates to control for confounding, especially by social support and functional status, are also adequate. The results of the study may become confirmatory if correlation of religious services with involvement of areas in the brain and the underlying causes are studied by positron emission tomography and functional magnetic resonance imaging. In a lon- gitudinal retrospective study involving 103 subjects in which fMRI study of brain was done, religion or spirituality, but not frequency of attendance, was associated with thicker cortices in the left and right parietal and occipital regions [51]. The mesial frontal lobe of the right hemisphere, and the cuneus and pre-cuneus in the left hemisphere were involved independent of familial risk.

The effects of importance on cortical thickness were significantly stronger in the high-risk than in the low-risk group, particularly along the mesial wall of the left hemisphere, in the same region where we previously reported a significant thinner cortex associated with a familial risk of developing depressive illness. It is possible that an association of thickness in brain cortex with a high importance of religion or spirituality may provide resilience to the production of depression among subjects with family history of major depression. This is possible due to expansion of cortical reserve which inhibits the vulnerability posed by cortical thinning predisposing familial depression [51]. New therapies targeting new imaging markers should be used for advancement of therapies and greater benefit to patients.

\section{Influence of Circadian Clock in the Brain on Circadian Rhythms}

All the physiological functions and metabolism are under influence of central circadian clock present in the supra-chiasmatic nucleus, situated at the lateral hypothalamus of the brain. Circadian patterns of cardiovascular vulnerability are well characterized, with a peakincidence of acute myocardial infarction and stroke secondary to atherothrombosis in the morning [59]. The circadian clock may thus take part in the pathological process of atherosclerosis induced by hyperlipidemia. Hyperlipidemia was shown to alter both the rhythmicity and expression of circadian genes in an atherosclerotic mouse model [59]. Diet-induced circadian disruption may thus affect the process of atherosclerosis and some acute cardiovascular consequences. Hypercholesterolemia has been associated with impaired fibrinolysis due to enhanced plasminogen activator inhibitor-1 (PAI-1) activity, which has also been implicated in atherosclerosis [30]. PAI-1's gene expression is coordinated under the circadian clock gene in the mouse heart and liver [60]. Although, hypercholesterolemia did not significantly affect the circadian expression of clock genes (Per2 and Bmal1) and clock-controlled genes (Dbp and E4bp4) in the liver, it enhanced the circadian rhythm of PAI-1 mRNA [30]. Restricted feeding for 4 hours during the day was shown to reset the gene expression of Per2, PAI-1, Nr4A1, and TNF区 [60]. The increased risk of acute atherothrombotic events in the morning may thus be augmented under conditions of hypercholesterolemia.

Intestinal circadian clock genes are synchronized by signals emanating from the suprachiasmatic nuclei (SCN) and from other environmental factors, such as food availability. In one study, lipid absorption was shown to depend on circadian rhythms 
coordinating microsomal triglyceride transfer protein, apoAIV, and nocturnin [61]. Disruptions in the sleep/wake cycle and irregularities in temporal food intake induce undesirable changes in intestinal function that predispose individuals to atherosclerosis and also to various metabolic disorders such as the metabolic syndrome, obesity, and diabetes [61]. Further evidence suggests that melatonin, a hormone produced primarily in the pineal, has positive effects on the cardiovascular system, which is modulated by the SCN [62]. For instance, patients with coronary artery disease have a low melatonin production rate [62]. Vascular melatoninergic receptors are thought to be functionally linked to vasoconstrictor or vasodilatory effects of melatonin. Melatonin may attenuate molecular and cellular damages resulting from cardiac ischemia/ reperfusion in which destructive free radicals are involved. Antiinflammatory and antioxidative properties of melatonin are also believed to be involved in the protection against atherosclerosis [62].

The brain helps us cope with adverse situations, influencing the activity of the adaptive endocrine, nervous and immune systems [63]. In the physiological response, hormones involved in the hypothalamic-pituitary-adrenal (HPA) network and their receptors are eminently circadian periodic, cortisol in particular $[64,65]$. The central role is played by hippocampus as an important connection between cortex and hypothalamus, in conjunction with the SCN coordinating the cortisol rhythm [63], with modulation by the pituitary and the pineal by feed sideward mechanisms $[64,65]$. Hyperactivity of the HPA network is a feature that can be present in chronic conditions, including atherosclerosis. Martocchia et al. [63] proposed that the different molecules interacting at the different sites of the HPA network, including agents acting on the SCN, offer novel therapeutic strategies to counteract hyperactivity of the HPA network in order to reduce the burden of disorders related to excessive loads, including atherosclerosis. In a cohort study, 114 adult offspring of depressed and nondepressed parents, were followed longitudinally for 20 years [66]. The effect was most pronounced among offspring at high risk for depression by virtue of having a depressed parent. Offspring who reported at year 10 that religion or spirituality was highly important to them, had about one-fourth the risk of experiencing major depression between years 10 and 20 compared with other participants. The protective effect was found primarily against recurrence rather than onset of depression. It is possible that a high self-report rating of the importance of religion or spirituality may have a protective effect against recurrence of depression, particularly in adults with a history of parental depression [66].

In another study, thicker cortex was associated with a high importance of religion or spirituality, which may confer resilience to the development of depressive illness in individuals at high familial risk for major depression. This may be possible by expanding a cortical reserve that counters to some extent the vulnerability that cortical thinning poses for developing familial depressive illness
$[67,68]$. There is a diminished production of melatonin at the very early stages of memory dysfunction [69]. The role of melatonin in the restorative value of sleep and its sleep-anticipating effects resulting in attenuated activation of certain brain networks are gaining a new perspective [69]. The role of poor sleep quality in the development of $\beta$ amyloid, particularly in the precuneus, is unravelled. More recently discovered relationship between circadian clock, sleep and neurodegeneration, indicate the use of melatonin for early intervention, to promote healthy physical and mental ageing. The sleep-wake cycle is the most overt circadian rhythm. Sleep is an orchestrated neurochemical process involving sleep-promoting and arousal centers in the brain.

Sleep appear to have a major role in the restoration of brain energy, in the switching off of external inputs and off-line processing of information acquired during wake, in the facilitation of the plasticity of cerebral changes that underlie learning, memory consolidation and extinction, and in the activation of the recently discovered lymphatic system that is responsible for brain metabolite clearance. These functions are critical for brain development, physical and mental health, and the maintenance of cognitive functions culminating in a sense of well-being and daytime vigilance. A study using PET, compared the differences in the brain glucose metabolism between morning and evening acquisitions, showing decreased metabolism at the cortical level in the temporal and occipital lobes [69].The evening quiet wakefulness was associated with greater metabolism in hypothalamic and brainstem structures, compared to morning. This finding putatively encompassed multiple sleep/wake or arousal promoting nuclei [70]. fMRI data obtained in resting state, found alterations in functional connectivity between the medial temporal lobe (MTL) and other areas in the brain that were observed in the morning and evening [70].

The local areas in the morning were functionally connected with bilateral MTL regions. However, the connectivity of these regions spreaded cortically in the evening, in multiple brain areas considered important for memory function. These results indicate that chronotherapy using new imaging markers can be used to develop new therapies in the treatment of diseases. Functional imaging studies of sleep deprivation have characterized its effects on a number of cognitive domains, the best studied of these being working memory $[69,70]$. There is a growing appreciation that it is important to consider inter-individual differences in vulnerability to sleep deprivation, task and task difficulty when interpreting imaging results. Our understanding of the role of sleep and the dynamic evolution of offline memory consolidation has benefited greatly from human imaging studies. Most prominently, bilateral medial temporal regions were locally connected in the morning but in the evening exhibited strong correlations with frontal and parietal brain regions involved in memory retrieval. Both hippocampal-dependent and hippocampal-independent memory systems have been studied. 
Functional imaging studies contrasting sleep-deprived and well-rested brains provide substantial evidence that sleep is highly important for optimal cognitive function and learning. The experimental paradigms developed to date merit evaluation in clinical settings to determine the impact of sleep disruption in sleep disorders. It is proposed, that circadian alterations in blood pressures and heart rate as well as in other physiological functions can be observed in brains SCN and lateral hypothalamus [71-74]. It seems that the influence of environment and religious services on neuro-cardiac systems can be examined by imaging studies to find new targets of therapies [75-77]. Since treating osteoporosis by bisphosphonates also modulate brain as well as CVDs, it appears that these agents provide benefit, at least in part via modulating new imaging markers of osteoporosis, atherosclerosis and brain degeneration which can be observed by new technologies of imaging [78-82].

\section{Conclusion}

In brief, fMRI which is a novel technology, may reveal altered structural as well as functional changes in the prefrontal-temporal communication by using diffusion tensor imaging (DTI) which provide evidence indicating myelin loss of white matter in schizophrenia. fMRI tracks several areas in the brain, in particular the amygdala and hypothalamus which are under the influence of psychosocial stress, including depression, that may predispose systemic inflammation, resulting in arterial inflammation and, in turn, atherosclerosis. fMRI studies indicate that the sleep quality appears to be under control of hippocampus. The effects of brain and body dysfunction appears to be bidirectional. In PET imaging, compared to the morning, evening quiet wakefulness may be related to greater rise in metabolism in hypothalamus and structures in the brain stem. This finding, putatively encompasses multiple nuclei concerned with sleep/wake or arousal. The changes and differences in functional connectivity measurements, between the medial temporal lobe and the rest of the brain were obtained in the morning and evening via fMRI studies. It is possible that religious services may be protective due to its association with thickening of the cortex in the brain. New therapies may be developed by using new neuroimaging markers via new technologies, for advancement of therapy of both psychological disorders as well as CVDs.

\section{Acknowledgement}

Acknowledgements are given to KEGA project, Ministry of Education, Slovak Republic, number 063UK-4/2017.

\section{References}

1. Abramova M, Chibisov S, Singh RB, Fedacko J, Shastun S, et al. (2017) Neuroanatomy and neurophysiology of coronary atherothrombosis. World Heart Jour 9(3): 197-204.

2. Galal NE, Hristova K, Singh RB, Pella D, olpindo MAS, et al. (2016) Cardiovascular Imaging: the future of cardiology investigation. World Heart J 8: 125-132.

3. Singh RB, Bjorklund G, Shastun S, Cornelissen G, Elkilany GN, et al. (2016) Mental and spritual health and the heart: a viewpoint. World Heart J 8(3): 233-238.
4. Tawakol A, Ishai A, Takx RAP, Figueroa AL, Ali A, et al. (2017) Relation between resting amygdalar activity and cardiovascular events: a longitudinal and cohort study. Lancet 389(10071): 834-845.

5. Singh RB, Torshin VI, Bawareed OA, Verma N, Mojto,V, et al. (2018) Physiological imaging; a new approach for assessment of neuropsychiatric and cardiovascular diseases. World Heart J 10:

6. Li S, Stampfer MJ, Williams DR, Tyler J (2016) Vander Weele TJ. Association of religious service attendance with mortality among women. JAMA Intern Med 176(6): 777-785.

7. Thackeray JT, Hupe HC, Wang Y, Bankstah JP, Berding G, et al. (2018) Myocardial inflammation predicts remodeling and neuro-inflammation after myocardial infarction J Am Coll Cardiol 71(3): 263-275.

8. Makovac E, Garfinkel S, Bassi A, Basile B, Macaluso E, et al. (2018) Fear processing is differentially affected by lateralized stimulation of carotid baroreceptors. Cortex 99: 200-212.

9. Zunhammer M, Bingel U, Wager TD (2018) Placebo effects on the neurologic pain signature; a meta-analysis of individual participant functional magnetic resonance imaging data. JAMA Neurol 75(11): 1321-1330.

10. Shaibani AA, Elkilany G, Singh RB (2015) Stress, peace and the heart. World Heart J 7: 265-274.

11. Nabi H, Kivimäki M, Batty GD, Shipley MJ, Britton A, et al. (2013) Increased risk of coronary heart disease among individuals reporting adverse impact of stress on their health: the Whitehall II prospective cohort study. Eur Heart J 34 (34): 2697-2705.

12. Rosengren A, Hawken S, Ounpuu S, Sliwa K, Zubaid M, et al. (2004) Association of psychosocial risk factors with risk of acute myocardial infarction in 11119 cases, 13648 controls from 52 countries (the INTERHEART Study): case control study. Lancet 364(9438): 953-962.

13. Xu T, Li W, Teo K, Wang XY, Liu LS, et al. (2011) Association of psychological risk factors and acute myocardial infarction in China: the INTERHEART China study. Chin Med J 124(14): 2083-2088.

14. Chauveau F, Lange MD, Jüngling K, Lesting J, Seidenbecher T, et al. (2012) Prevention of stress-impaired fear extinction through neuropeptide $\mathrm{s}$ action in the lateral amygdala. Neuropsycho-pharmacology 37(7): 15881599.

15. Phan KL, Wager T, Taylor SF, Liberzon I (2002) Functional neuroanatomy of emotion: a meta-analysis of emotion activation studies in PET and fMRI. Neuroimage 16(2): 331-348.

16. Abumaria N, Yin B, Zhang L, Li XY, Chen T, et al. (2011) Effects of elevation of brain magnesium on fear conditioning, fear extinction, and synaptic plasticity in the infralimbic prefrontal cortex and lateral amygdala. J Neurosci 31(42): 14871-14881.

17. Ressler KJ (2010) Amygdala activity, fear, and anxiety: modulation by stress. Biol Psychiatry 67(12): 1117-1119.

18. Aschbacher K, Kornfeld S, Picard M, Puterman E, Havel PJ, et al. (2014) Chronic stress increases vulnerability to diet-related abdominal fat, oxidative stress, and metabolic risk. Psychoneuroendocrinology 46: 1422.

19. Lagraauw HM, Kuiper J, Bot I (2015) Acute and chronic psychological stress as risk factors for cardiovascular disease: Insights gained from epidemiological, clinical and experimental studies. Brain Behav Immun 50: 18-30.

20. Mier W, Mier D (2015) Advantages in functional imaging of the brain. Frontiers in Human Neuroscience 9: 249.

21. Logothetis NK, Pauls J, Augath M, Trinath T, Oeltermann A (2001) Neurophysiological investigation of the basis of the fMRI signal. Nature 412(6843): 150-157

22. Raichle ME (2001) Cognitive neuroscience. Bold insights. Nature 412(6843): 128-130

23. Shenton ME, Dickey CC, Frumin M, McCarley RW (2001) A review of MRI 
findings in schizophrenia. Schizophr. Res 49(1-2): 1-52.

24. Kindermann SS, Karimi A, Symonds L, Brown GG, Jeste DV (1997) Review of functional magnetic resonance imaging in schizophrenia. Schizophr Res 27(2-3): 143-156.

25. Zhou Y, Liang M, Jiang T, Tian L, Liu Y, et al. (2007) Functional dysconnectivity of the dorsolateral prefrontal cortex in first-episode schizophrenia using resting-state fMRI. Neurosci. Lett 417(3): 297-302.

26. Meyer Lindenberg A, Poline JB, Kohn PD, Holt JL, Egan MF, et al. (2001) Evidence for abnormal cortical functional connectivity during working memory in schizophrenia. Am J Psychiatry 158(11): 1809-1817.

27. Burns J, Job D, Bastin ME, Whalley H, Macgillivray T, et al. (2003) Structural disconnectivity in schizophrenia: a diffusion tensor magnetic resonance imaging study. Br J Psychiatry 182: 439-443.

28. Mier D, Kirsch P, Meyer Lindenberg A (2010) Neural substrates of pleiotropic action of genetic variation in COMT: a meta-analysis. Mol Psychiatry 15(9): 918-927.

29. Poels EM, Kegeles LS, Kantrowitz JT, Javitt DC, Lieberman JA, et al. (2014) Glutamatergic abnormalities in schizophrenia: a review of proton MRS findings. Schizophr Res 152(2-3): 325-332.

30. Nordberg A, Rinne J0, Kadir, Långström B (2009) The use of PET in Alzheimer disease. Nat. Rev Neurol 6(2): 78-87.

31. Bucerius J, Manka C, Schmaljohann J, Mani V, Gündisch D, et al. (2012) Feasibility of [18F]-2-Fluoro-A85380-PET imaging of human vascular nicotinic acetylcholine receptors in vivo. JACC Cardiovasc. Imaging 5(5): 528-536.

32. Rotella F, Mannucci E (2013) Depression as a risk factor for diabetes: a meta-analysis of longitudinal studies. J Clin Psychiatry 74 (1): 31-37.

33. Valkanova V, Ebmeier KP (2013) Vascular risk factors and depression in later life: a systematic review and meta-analysis. Biol Psychiatry 73(5): 406-413.

34. Koenigs M, Grafman J (2009) The functional neuroanatomy of depression: distinct roles for ventromedial and dorsolateral prefrontal cortex. Behav Brain Res 201(2): 239-243.

35. Duivis HE, Vogelzangs N, Kupper N, de Jonge P, Penninx BW (2013) Differential association of somatic and cognitive symptoms of depression and anxiety with inflammation: findings from the Netherlands Study of Depression and anxiety (NESDA). Psychoneuro endocrinology 38(9): 1573-1585.

36. Fagundes CP, Glaser R, Hwang BS, Malarkey WB, Kiecolt Glaser JK (2013) Depressive symptoms enhance stress-induced inflammatory responses. Brain Behav Immun 31: 172-176.

37. Chaves H, Pella D, Singh RB, Saboo B, Hristova K, et al. (2016) The challenges of prevention of cardiovascular diseases. A scientific statement of the International College of Cardiology. World Heart J 8(4): 282-288.

38. Seaman DR (2002) The diet-induced pro-inflammatory state: a cause of chronic pain and other degenerative diseases? J Manipul Physiol Ther 25(3): 168-179.

39. Patriquin MA, Mathew SJ (2017) The Neurobiological Mechanisms of Generalized Anxiety Disorder and Chronic Stress. Chronic stress 1: 1-10.

40. Murphy TM, O’Donovan A, Mullins N, O’Farrelly C, McCann A, et al. (2015) Anxiety is associated with higher levels of global DNA methylation and altered expression of epigenetic and interleukin- 6 genes. Psychiatr Genet 25(2): 71-78.

41. Makovac E, Meeten F, Watson DR, Herman A, Garfinkel SN, et al. (2016) Alterations in amygdala-prefrontal functional connectivity account for excessive worry and autonomic dysregulation in generalized anxiety disorder. Biol Psychiatry 80(10): 786-795.

42. Fonzo GA, Etkin A (2016) Brain connectivity reflects mental and physica states in generalized anxiety disorder. Biol Psychiatry 80(10): 733-735.
43. Hilbert K, Lueken U, Beesdo Baum K (2014) Neural structures, functioning and connectivity in generalized anxiety disorder and interaction with neuroendocrine systems: a systematic review. J Affect Disord 158: 114-126

44. Etkin A, Prater KE, Schatzberg AF, Menon V, Greicius MD (2009) Disrupted amygdalar subregion functional connectivity and evidence of a compensatory network in generalized anxiety disorder. Arch Gen Psychiatry 66(12): 1361-1372.

45. Monk C, Telzer EH, Mogg K, Brendan P Bradley, Xiaoqin Mai, et al. (2008) Amygdala and ventrolateral prefrontal cortex activation to masked angry faces in children and adolescents with generalized anxiety disorder. Arch Gen Psychiatry 65(5): 568-576.

46. Roy AK, Fudge JL, Kelly C, Justin SA Perry, Teresa Daniele, et al. (2013) Intrinsic functional connectivity of amygdala-based networks in adolescent generalized anxiety disorder. J Am Acad Child Adolesc Psychiatry 52(3): 290-299.

47. Diekhof EK, Geier K, Falkai P, Gruber O (2011) Fear is only as deep as the mind allows: a coordinate-based meta-analysis of neuroimaging studies on the regulation of negative affect. Neuroimage 58(1): 275-285.

48. Maslowsky J, Mogg K, Bradley BP, Erin McClure Tone, Monique Ernst, et al. (2010) A preliminary investigation of neural correlates of treatment in adolescents with generalized anxiety disorder. J Child Adolesc Psychopharmacol 20(2): 105-111.

49. Pittig A, Arch JJ, Lam CW, Craske MG (2013) Heart rate and heart rate variability in panic, social anxiety, obsessive, Ä00EC; compulsive, and generalized anxiety disorders at baseline and in response to relaxation and hyperventilation. Int J Psychophysiol 87(1): 19-27.

50. Bernardi I, Wdowczyc Szulc J, Valenti C, Castoldi S, Passino C, et al. (2000) Effects of controlled breathing, mental activity and mental stress with or without verbalization on heart rate variability. J Am Coll Cardiol 35(6): 1462-1469.

51. Paul Labrador M, Polk D, Dwyer JH, Velasquez I, Nidich S, et al. (2006) Effects of a randomized controlled trial of transcendental meditation on components of the metabolic syndrome in subjects with coronary heart disease. Arch Intern Med 166(11): 1218-1224.

52. Miller L, Bansal R, Wickramaratne P, Hao X, Tenke CE, et al. (2014) Neuroanatomical Correlates of Religiosity and Spirituality: A Study in Adults at High and Low Familial Risk for Depression. JAMA psychiatry 71(2): 128-135.

53. Singh RB, Cornelissen G, Otsuka K, Juneja L, Halberg F (2012) Coronary risk factors and ambulatory blood pressure and heart rate in Asian Indians. The Open Nutraceuticals Journal 5 (Suppl 1-M5): 79-80.

54. Singh S, Singh G, Kartikey K, Singh RB (2009) Effect of pranayama breathing patterns on naso-bronchial diseases: The power of breath. In Handbook of Pulmonary Medicine, Nova Science Publishers NY 1-9.

55. Halberg F, Cornelissen G, Sothern RB, Czaplicki J, Schwartzkopff O (2010) Thirty-five-year climatic cycle in heliogeophysics, psycho- physiology, military politics, and economics. Izv Atmos Ocean Phy 46(7): 844-864.

56. Cornelissen G, Halberg F, Breus T, Syutkina EV, Baevsky R, et al. (2002) Non-photic solar associations of heart rate variability and myocardial infarction. J Atmos Solar-Terr Phys 64: 707-720.

57. Singh RB, Cornelissen G, Kumar A, Bathina S, Halberg F (2009) Larger circadian amplitude of heart rate associated with active prayer in Hindu Indians in Asia. World Heart J 2009 1: 219-222.

58. Hansson GK, Libby P, Tabas I (2015) Inflammation and plaque vulnerability. J Intern Med 278(5): 483-493.

59. Heidt T, Sager HB, Courties G, Dutta P, Iwamoto Y, et al. (2014) Chronic variable stress activates hematopoietic stem cells. Nat Med 20(7): 754758.

60. Hou L, Lu C, Huang Y, Chen S, Hua L, et al. (2009) Effect of hyperlipidemia on the expression of circadian genes in apolipoprotein $\mathrm{E}$ knock-out atherosclerotic mice. Lipids in Health \& Disease 8: 60. 
61. Kudo T, Nakayama E, Suzuki S, Akiyama M, Shibata S (2004) Cholesterol diet enhances daily rhythm of Pai-1 mRNA in the mouse liver. Am J Physiology - Endocrinology \& Metabolism 287(4): E644-E651.

62. Hussain MM, Pan X (2015) Circadian regulators of intestinal lipid absorption. J Lipid Res 56(4): 761-770.

63. Dominguez Rodriguez A, Abreu Gonzalez P, Reiter RJ (2009) Clinical aspects of melatonin in the acute coronary syndrome. Current Vascular Pharmacology 7(3): 367-373.

64. Martocchia A, Curto M, Toussan L, Stefanelli M, Falaschi P (2011) Pharmacological strategies against glucocorticoid-mediated brain damage during chronic disorders. Recent Patents on CNS Drug Discovery 6(3): 196-204.

65. Cornelissen G, Halberg F (1994) Introduction to Chronobiology. Medtronic Chronobiology Seminar, p. 52.

66. Halberg F, Guillaume F, Sanchez de la Peña S, Cavallini M, Cornelissen G (1986) Cephalo-adrenal interactions in the broader context of pragmatic and theoretical rhythm models. Chronobiologia 13(2): 137-154.

67. Miller L, Wickramaratne P, Gameroff MJ, Sage M, Tenke CE, et al. (2012) Religiosity and major depression in adults at high risk: a ten-year prospective study. Am J Psychiatry 169(1): 89-94.

68. Miller L, Bansal R, Wickramaratne P, Hao X, Tenke CE, et al. (2014) Neuroanatomical correlates of religiosity and spirituality: A study in adults at high and low familial risk for depression. JAMA psychiatry 71(2): 128-135.

69. Vlasova RM, Siddarth P, Krause B, Leaver AM, Laird KT, et al. (2018) Resilience and white matter integrity in geriatric depression. The American Journal of Geriatric Psychiatry 26(8): 874-883.

70. Zisapel N (2018) New perspectives on the role of melatonin in human sleep, circadian rhythms and their regulation. Brit J pharmacol 175(6): 3190-3199.

71. Shannon BJ, Dosenbach RA, Su Y, AG Vlassenko, LJ Larson Prior, et al. (2013) Morning-evening variation in human brain metabolism and memory circuits. Journal of Neurophysiology 109(5): 1444-1456.

\section{ISSN: 2574-1241}

DOI: 10.26717.BJSTR.2019.14.002484

Viliam Mojto. Biomed J Sci \& Tech Res

(C) This work is licensed under Creative

Submission Link: https://biomedres.us/submit-manuscript.php
72. Blagonravov ML, Frolov VA, Azova MM, Goryachev VA (2013) Characteristics of circadian rhythm of blood pressure during long-term hypertension development in SHR rats. Bull Exp Biol Med 155(5): 612614.

73. Blagonravov ML, Frolov VA, Goryachev VA, Azova MM (2015) Features of the structure of the circadian rhythm of blood pressure and heart rate under genetically determined hypertension in the experiment. Bull Exp Biol Med 158(6): 723-725.

74. Singh RB, Hristova K, Gligorijevic T, De Meester F, Saboo B, et al. (2015) Can circadian restriction of feeding modulate autonomic nervous system dysfunction and cardiometabolic risk? World Heart J 7(1): 31-42.

75. Singh RB, Anjum B, Garg R, Verma NS, Singh R, et al. (2012) Association of circadian disruption of sleep and night shift work with risk of cardiovascular disease. World Heart J 4(1): 23-32.

76. Mojto V, Singh RB, Chibisov S, Abramova M, Verma NS, et al. (2017) Can climate, weather, cosmos and environmental degradation predispose cardiovascular diseases and other diseases? World heart J 9(4): 333348.

77. Singh RB, Bjorklund G, Shastun S, Cornelissen G, Elkilany GN, et al. (2016) Mental and spritual health and the heart: a viewpoint. World Heart J 8(3): 233-238.

78. Gaemperli O, Delgado V, Habib G, Kaufmann PA, Bax JJ (2016) The year in cardiology 2015: imaging. Euro heart J.

79. Sing CW, Wong AYS, Kiel DP, Cheung EY, Lam JK, et al. (2018) Association of alendronate and risk of cardiovascular events in patients with hip fracture. J Bone Miner Res 33:1422-1433.

80. Abbasi J (2018) Amid osteoporosis treatment crisis, experts suggest addressing patients' bisphosphonate concerns. JAMA 319(24): 24642466.

81. Kartikey K, Singh G, Singh RB, Al-bawareed OA (2018) Bisphosphonates; osteoporosis and cardiovascular diseases. World Heart J 10: in press

82. Zameer S, Najmi AK, Vohra D, Akhtar M (2018) Bisphosphonates: Future perspective for neurological disorders. Pharmacological Reports 70: 900-907.

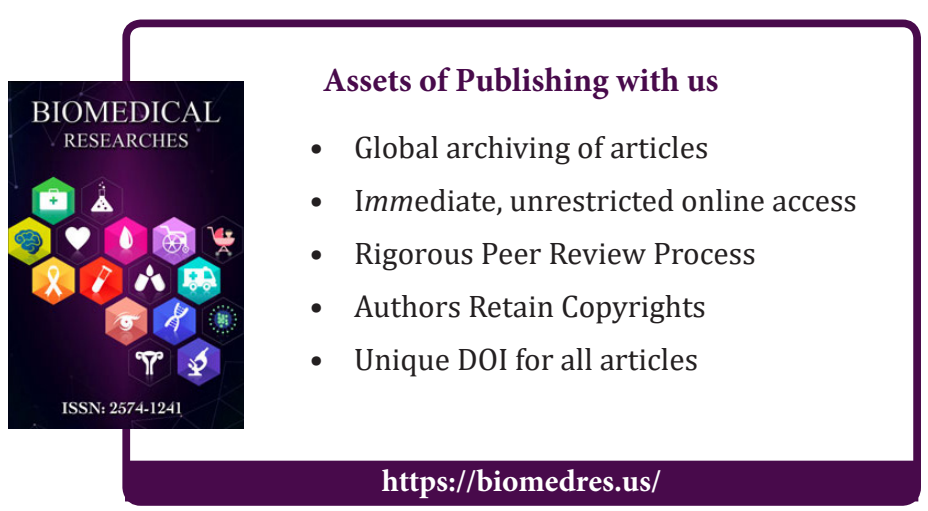

